\title{
RENTABILIDAD DEL SISTEMA DE ABONERA EN EL LITORAL ATLÁNTICO DE HONDURAS1
}

\author{
Gustavo Sain ${ }^{2}$, Ignacio Ponce ${ }^{3}$, Erik Borbón ${ }^{2}$
}

\begin{abstract}
RESUMEN
Rentabilidad del sistema de abonera en el Litoral Atlántico de Honduras. El Litoral Atlántico de Honduras se ha difundido un sistema de rotación de maíz con Mucuna s. p. conocido por los agricultores con el nombre de abonera. Debido a que poco se conoce sobre los factores que influyen en el proceso de adopción de esta práctica se planteó el presente estudio con el propósito de identificar estos factores, asícomo determinar la rentabilidad del sistema tanto en el corto como en el largo plazo y a la vez establecer los factores y mecanismos que afectan esta rentabilidad. El estudio se llevó a cabo en el departamento de Atlántida y comprende parte del municipio de La Ceiba y los municipios de Jutiapa, San Francisco y Tela. Para evaluar la rentabilidad se empleó como herramienta una expansión del análisis de presuppuesto parcial y análisis incremental para un periodo para tener un cuenta los efectos a través del tiempo. Para el análisis se tomó seis años como la vida media de la abonera. Al evaluar económicamente el sistema se encontró que el éste resulta más rentable que el tradicional a partir del segundo año en adelante. Si el criterio de rentabilidad es el retorno a la mano de obra familiar, el sistema de abonera también resulta más rentable a partir del segundo año y así permanece por todo el periodo.
\end{abstract}

\begin{abstract}
Profitability of a corn rotation system with Mucuna sp. in Honduras. A rotation system of corn (Zea mays L.) with Mucuna sp. has spread along the Atlantic Littoral of Honduras, known by the farmers as "abonera". Due that there is little known about the factors intluencing the adoption process, the present study was eondueted to identify them, as well as to determine the profitability of the system in the short and the long run and to set up the factors and mechanisms affecting this profitability. The study was conducted in the Department of Atlantida and comprises part of the Distric of La Ceiba and the Districs of Jutiapa, San Francisco and Tela. An expansion of the partial budget analysis and an incremental analysis for a period, in order to account for the effects along the time, were used as tools for evaluating its profitability. Six years were taken, for the analysis, as the average life span of the "abonera". It was found in the economical evaluation that the "abonera" is more profitable than the traditional system from the second year onwards. If the profitability approach is the return of the family hand labor, the "abonera" system is al so more profitable starting at the second year and remains so for the rest of the periodo.
\end{abstract}

\section{INTRODUCCION}

En el Litoral Atlántico de Honduras (Figura 1), la producción agrícola ocurre en dos épocas bien definidas: la primera, también llamada de primavera y la postrera o de verano. En gran medida la capacidad para producir en cada época está determinada por el régimen estacional de lluvias predominantes. En el Litoral Atlántico, el régimen de lluvias es tal que permite la siembra y cultivo de ambas estaciones con diferentes grados de éxito (Figura 2). Las lluvias inician su ciclo ascendente en el mes de mayo y continuan en ascenso hasta llegar a noviembre que es el mes más lluvioso del año. A partir de allí comienzan su fase descendente hasta llegar al mes de abril. Los ciclos productivos de primera y postrera se acomodan a este régimen. La siembra de primera del maíz comienza generalmente en junio-julio para ser cosechado en setiembre-octubre, mientras que la siembra de postrera se

\footnotetext{
1 Presentado en la XXXIX Reunión Anual del PCCMCA en Guatemala, América Central. 28 de marzo - 3 de abril, 1993.

2 Economistas en el Centro Internacional de Mejoramiento de Maíz y Trigo con sede en San José, Costa Rica.

3 Economista en la Secretaría de Recursos Naturales de Honduras con sede en San Pedro Sula.
} 




Figura 1. Localización del Departamento de Atlántida y del Area de Estudio.

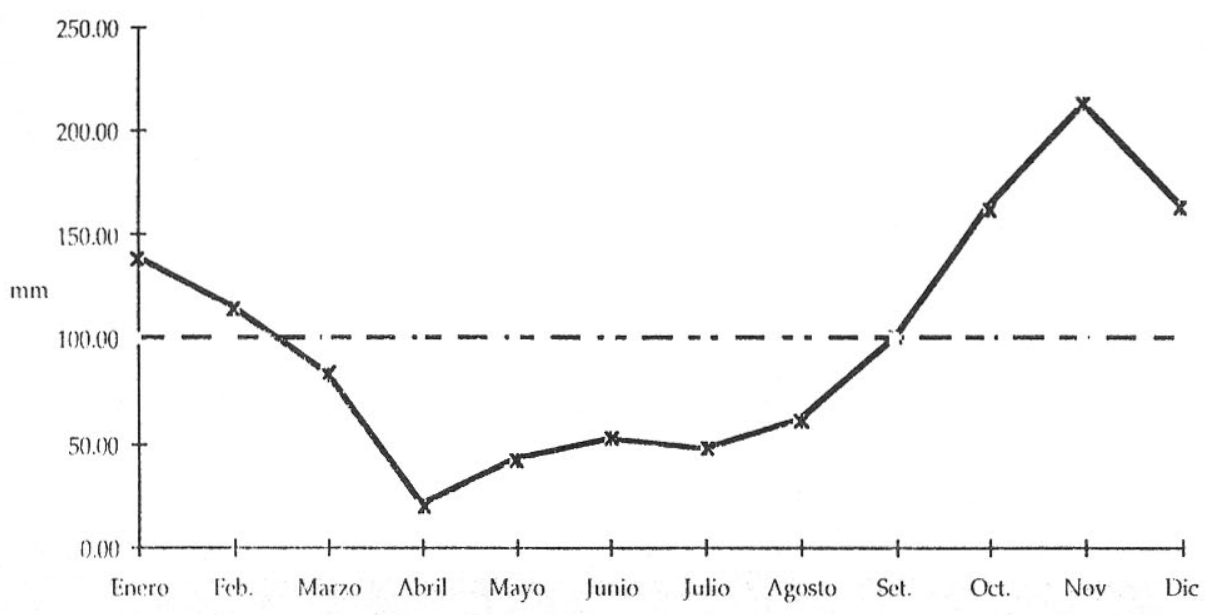

Figura 2. Patron estacional de las lluvias en el ! itoral Atlántico de Honduras (1972-1990). 
realiza en diciembre-enero para ser cosechado en abrilmayo.

La estación de primera o primavera fue tradicionalmente la más importante en lo que a prpducción de maíz en el Litoral Atlántico se refiere. A comienzo de la década de los 80 e $165 \%$ de la producción total de la región se producía en primera, sin embargo, la situación se ha revertido y a que la participación relativa de la postrera en la producción ha crecido en los últimos 15 años a razón de casi $1 \%$ anual hasta alcanzar más del 50\% de la producción total en las últimas tres campañas (Figura 3).

Aúnque ambas épocas tienen a juicio de los agricultores, ventajas y desventajas para la producción de maíz, la siembra de postrera presenta dos ventajas bien definidas. En primer lugar la cosecha de postrera ocurre en los meses más secos del año (abril-mayo) por lo cual el maíz tiene una mejor sanidad que en primera donde se cosecha con mucha lluvia y existe el problema de pudrición de mazorca. Una segunda ventaja de la siembra de postrera es que permite aprovechar el marcado ciclo estacional que se observan en los precios de venta del maíz y la cosecha ocurre cuando los precios están por arriba del promedio anual.

El aumento de la importancia relativa de la producción de postrera se debe principalmente al incremento del área cultivada el cual está asociado a la difusión de la siembra de maíz con frijol de abono (Mucuna aterrima sp.) en el sistema llamado "abonera." El uso del frijol de abono muestra un significativo incremento en el área en la década de los 80 y su difusión ha sido recientemente sujeto de estudio de varios trabajos (A vila y López, Buckles et al., 1991, 1992). Mientras que en 1980 menos del 5\% de los agricultores utilizaban el sistema, en 1989, este porcentaje superaba el $60 \%$. La curva de difussion del sistema de abonera en el Dpto. de Atlántida mostró que entre 1981 y 1990 la difusión fue casi lineal a una tasa de crecimiento anual de casi $7 \%$ (Buckles et al., 1992).

Varias ventajas han sido asociadas al sistema de abonera por los agricultores (Flores y Licona, Avila y López, Buckles et al). Entre ellas se pueden mencionar: 1) El ahorro de mano de obra en la preparación del terreno para la siembra. 2) Un ahorro de mano de obra en el control de malezas, ya que el frijol de abono disminub la incidencia de éstas; y 3) Un ahorro en capital de trabajo en términos de fertilizantes, ya que el maíz en abonera no necesitaba la aplicación de fertilizantes inorgánicos para obtener el mismo rendimiento que con la aplicación de este tipo de fertilizantes. Este efecto también puede ser visto como un incremento en los beneficios brutos por aumento en los rendimientos respecto al maíz cultivado sin abonera y sin fertilización inorgánica.

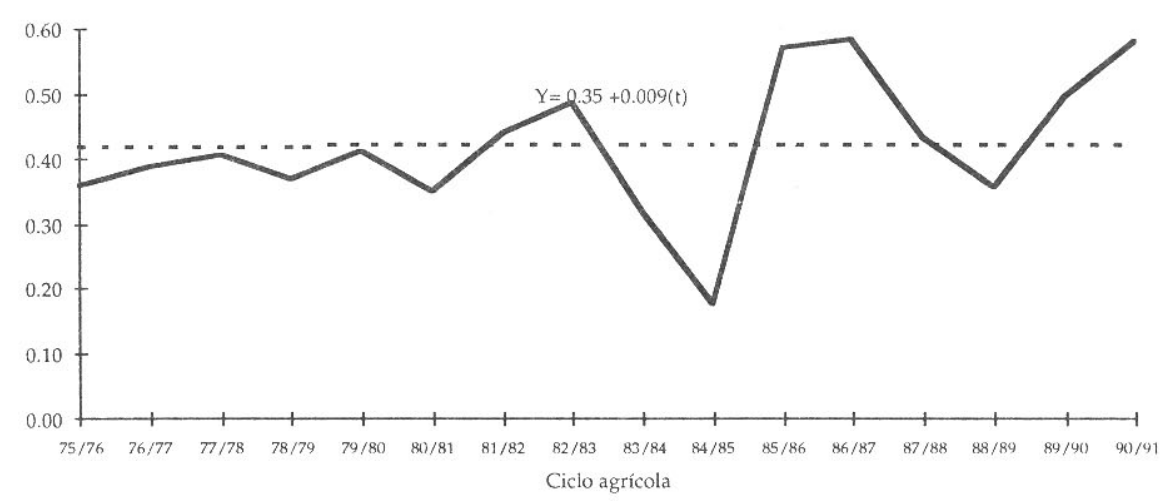

Figura 3. Evolución y tendencia de la importancia relativa de la superficie cultivada con maíz en postrera en el Litoral Atlántico de Honduras, 75/76-90/91. 
De acuerdo entonces a la percepción de los agricultores el efecto neto del sistema de abonera es de una reducción en los costos de operación, al menos cuando la abonera ya está implantada. Esta reducción se realiza esencialmente porun ahorro de mano de obra necesaria para la preparación del terreno (chapia) y control de malezas.

Adicionalmente el uso del frijol de abono a nivel del sistema de finca permite el incremento del área en la siembra de postrera lo que permite al agricultor aprovechar las ventajas ya mencionadas de cosechar maíz en una época donde la precipitaciónes son mínimas y los precios de maíz son estacionalmente altos.

No cabe duda que la rentabilidad de corto y largo plazo del sistema en relación con el uso alternativo del suelo juega un papel importante en la decisión de adoptar una práctica de conservación del suelo (Anderson y Thampapillai, Napier). En este trabajo se estima la rentabilidad del sistema de abonera en relación al sistema tradicional, tanto en el corto como en el largo plazo; y se analizan los factores y mecanismos que afectan esta rentabilidad, en especial el impacto que la adopción del sistema de abonera tiene sobre el uso de mano de obra.

El trabajo se localiza en los municipios de La Ceiba, Jutiapa, San Francisco y Tela pertenecientes al Departamento de Atlántida. El Departamento de Atlántida es uno de los tres Departamentos que forman la región denominada el Litoral Atlántico de Honduras (Figura 1).

\section{MATERIALES Y METODOS}

\section{EL SISTEMA DE PRODUCCION DE MAIZ: Tamaño de finca, uso de la tierra y el sistema de abonera}

En el Litoral Atlántico predominan fincas de tamaño pequeño siendo la mayoría de los agricultores dueños de la finca. El $74 \%$ de la población de agricultores productores de maíz en el Litoral Atlántico de Honduras poseen alguna de las parcelas que trabajan en propiedad pudiendo en caso de necesitar alquilar tierras a terceros para su explotación. Los agricultores que poseen tierras, tienen un tamaño de finca promedio de 11 has, mientras que aquellos que no poseen tierra, el tamaño promedio de la finca es de dos has.

Aproximadamente el $30 \%$ de la superficie de unafinca típica o modal de aquellos agricultores que son dueños de sus tierras, se destina a los cultivos mientras que el restante $70 \%$ se destina a pastos y a descanso (guamil). El cultivo más importante tanto en primavera como en postrera es el maíz, pero también se cultiva arroz, y frijol en diferentes épocas del año (Cuadro 1). Además de las actividades realizadas en tierra propia los agricultores pueden alquilar tierra a terceros para realizar alguna actividad. Esta modalidad se da principalmente con el maíz de postrera donde la mitad de los agricultores alquilan una manzana adicional para su siembra. Este comportamiento podría explicar, al menos parcialmente, el incremento de las siembras de postrera y la difusion de aboneras ya que podría pensarse que los agricultores implantan la abonera en parcelas propias y siembran una o mas parcelas adicionales bajo el sistema tradicional en tierras prestadas o alquiladas.

Otros cultivos como yuca, plátanos, etc. usualmente son sembrados en el solar y destinados al autoconsumo. Casi la totalidad de los agricultores mantienen gallinas y la mayoría bestias de carga destinadas a transportar la producción de las parcelas hacia la casa o hacia el lugar de venta.

La siembra de maíz en abonera es un sistema de rotación del maíz de postrera con el frijol de abono. Para implantar una abonera se siembra el frijol de abono en forma intercalada alrededor de los 40 a 50 días depués de la siembra del maíz de postrera. Este se cosecha entre marzo y abril dejando el frijol de abono que se desarrolle hasta fimes de noviembre cuando produce semilla. En ese momento el agricultor comienza la preparación de la siembra del maíz chapeando la mucuna y sembrando el maíz a chuzo unas semanas más tarde. Este ciclo se repite por varios años. La Figura 4 muestra la distribución de frecuencia de la edad de las aboneras en el Litoral Atlántico como así también los parámetros de esa 
Cuadro 1. Uso de la tierra de acuerdo al sistema. Agricultores con tierra. Litoral Atlántico de Honduras.

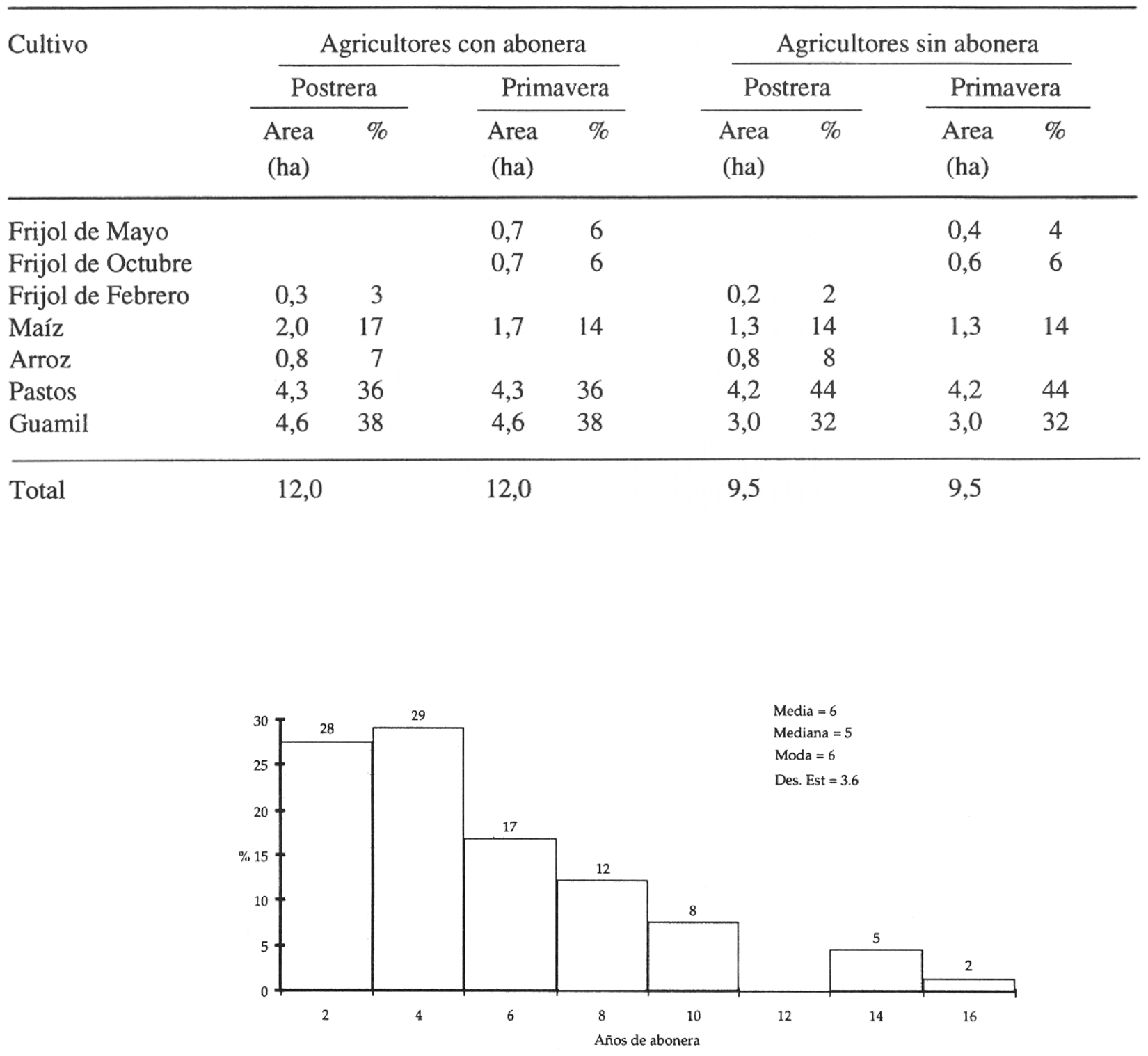

Figura 4. Distribución de la edad de las aboneras en el Litoral Atlántico de Honduras. 1991.

distribución. Tal como era de esperar, la distribución de esta variable se concentra a la izquierda de la edad promedio de seis años, lo que indica que la mayoría de las aboneras son jóvenes respecto al promedio, reflejando la tendencia expansiva del fenómeno.

El $71 \%$ de los agricultores propietarios siembran maíz en postrera bajo el sistema de aboneras mientras que solo $29 \%$ de ellos los hacen bajo el sistema tradicional.
Tal como es de esperarse en caso de tecnologías de conservación, el $87 \%$ de aquellos que siembran en abonera lo hacen en terrenos propios.

Si la disponibilidad de tierra fuera un factor limitante, el sistema de abonera y el tradicional deben verse como mutualmente excluyentes . Es decir, el agricultor puede usar la parcela bajo el sistema tradicional por tres ciclos (un año y medio) y luego dejada en descanso por 4 años 
y medio o alternativamente implantar un sistema de abonera por ese mismo período.

Sin embargo la evidencia encontrada en el Litoral Atlántico, indica que éste no es el caso. Por un lado existe alrededor de un $35 \%$ del total de la tieITa en el sistema de finca típico del agricultor que se encuentra en descanso. Además la expansión del sistema de abonera se ha realizado en gran medidad mediante la expansión del área sembrada y no mediante sustitución del área sembrada. El número de parcelas de maíz sembradas en postrera fué significativamente superior en el grupo de agricultores propietarios que sembraron al menos una parcela en abonera que en aquellos que se mantuvieron sin embrar maíz en abonera, Cuadro 2. También se encontró que la superficie sembrada con maíz en postrera aumentó significativamente en el grupo de agricultores que tenían abonera, respecto a aquellos que no habían adoptado el sistema, Cuadro 3. Tal como era de esperar no se encontró evidencia concluyente que la presencia o no de la abonera afectara en forma significativa el número de parcelas ni la superficie sembradas con maíz en primavera.

Es decir que a nivel de finca, la incorporación del sistema de abonera en el sistema ha permitido aumentar el área cultivada con maíz en postrera a mediante la incorporación de una parcela adicional. El Cuadro 4 muestra en forma esquemática como afecta al uso de la tierra la incorporación de la abonera. Al cabo del ciclo de seis años, en el sistema tradicional se han cultivado 12 unidades (manzanas, has etc) de maíz utilizando cuatro parcelas, en el sistema de abonera en ese mismo período se cultivan 18 unidades (un aumento del 50\%) utilizando solo 5 parcelas (un aumento del 25\%). La incorporación de la abonera permite seis cosechas de maíz adicionales usando solo una parcela más. Notesé que en el caso del sistema de abonera existirán dos parcelas de maíz de postrera y solo una de primavera, tal como lo confirman los datos encontrados en la encuesta (Cuadro 2).

\section{RESULTADOS Y DISCUSION}

\section{LA RENTABILIDAD DEL SISTEMA DE ABONERA}

El análisis económico del sistema de abonera parte del manejo que el agricultor realiza del sistema tal como se ilustrara en el Cuadro 4, en donde se incorpora una parcela adicional para se mbrarla bajo el sitema de abonera. En este caso se considera que el uso alternativo de esta parcela adicional sería intervenir como una parcela más en una rotación tradicional, por ejemplo como parcela No 1 en el Cuadro 4.

Bajo esta circunstancia, el uso de la parcela en el sistema tradicional genera un flujo de costos y beneficios durante los tres primeros ciclos debido al cultivo del maíz y un monto final al término del período debido a la

Cuadro 2. Número de parcelas de maíz sembradas en postrera y primavera de acuerdo al sistema. Agricultores con tierra. Litoral Atlántico de Honduras.

\begin{tabular}{|c|c|c|c|c|}
\hline & \multicolumn{2}{|c|}{ Postrera } & \multicolumn{2}{|c|}{ Primera } \\
\hline & $\begin{array}{l}\text { Agricultores } \\
\text { con abonera }\end{array}$ & $\begin{array}{c}\text { Agricultores } \\
\text { sin abonera }\end{array}$ & $\begin{array}{l}\text { Agricultores } \\
\text { con abonera }\end{array}$ & $\begin{array}{c}\text { Agricultores } \\
\text { sin abonera }\end{array}$ \\
\hline Media & 1,8 & 1,3 & 1,3 & 1,3 \\
\hline Moda & 2 & 1 & 1 & 1 \\
\hline Desviación Estandard & 0,9 & 0,7 & 0,6 & 0,8 \\
\hline Prob $(T<=t)$ una cola. & & & & \\
\hline $\begin{array}{l}\text { Ho: No con abon. }>=\text { No sin } \\
\text { abon. }\end{array}$ & \multicolumn{2}{|c|}{0,008} & 0,45 & \\
\hline
\end{tabular}


Cuadro 3. Superficie sembrada con maíz en postrera y primavera de a cuerdo al sistema. Agricultores con tierra. Litoral Atlántico de Honduras.

\begin{tabular}{lccccc}
\hline & \multicolumn{2}{c}{ Postrera } & & \multicolumn{2}{c}{ Primera } \\
\cline { 2 - 3 } \cline { 5 - 6 } & $\begin{array}{c}\text { Agricultores } \\
\text { con abonera }\end{array}$ & $\begin{array}{c}\text { Agricultores } \\
\text { sin abonera }\end{array}$ & $\begin{array}{l}\text { Agricultores } \\
\text { con abonera }\end{array}$ & $\begin{array}{c}\text { Agricultores } \\
\text { sin abonera }\end{array}$ \\
\hline Media & 2,6 & 1,7 & & 2,1 & 1,6 \\
Moda & 2 & 1 & & 2 & 1 \\
Desviación Estandard & 2,7 & 1,2 & & 2,1 & 1,3 \\
Prob $(\mathrm{T}<=\mathrm{t})$ una cola. & & 0,03 & & & 0,13 \\
Ho: No con abon. $>=$ No sin abon. & & & & & \\
\hline
\end{tabular}

Cuadro 4 . El uso de la tierra bajo los dos sistemas de cultivo.

\begin{tabular}{|c|c|c|c|c|c|c|c|c|c|c|}
\hline \multirow[b]{3}{*}{ Año } & \multirow[b]{3}{*}{ Epoca } & \multicolumn{8}{|c|}{ Sistema } & \multirow[b]{3}{*}{5} \\
\hline & & \multicolumn{4}{|c|}{$\begin{array}{c}\text { Tradicional } \\
\text { Número de parcela: }\end{array}$} & \multicolumn{4}{|c|}{$\begin{array}{c}\text { Abonera } \\
\text { Número de parcela: }\end{array}$} & \\
\hline & & 1 & 2 & 3 & 4 & 1 & 2 & 3 & 4 & \\
\hline \multirow[t]{2}{*}{1} & Primera & M & & & & M & & & & \\
\hline & Postrera & $\mathrm{M}$ & & & & M & & & & $\mathrm{M}$ \\
\hline \multirow[t]{2}{*}{2} & Primera & M & & & & $\mathrm{M}$ & & & & A \\
\hline & Postrera & & $\mathrm{M}$ & & & & M & & & M \\
\hline \multirow[t]{2}{*}{3} & Primera & & M & & & & M & & & A \\
\hline & Postrera & & M & & & & $\mathrm{M}$ & & & M \\
\hline \multirow[t]{2}{*}{4} & Primera & & & M & & & & $\mathrm{M}$ & & A \\
\hline & Postrera & & & M & & & & $\mathrm{M}$ & & M \\
\hline \multirow{2}{*}{5} & Primera & & & $\mathrm{M}$ & & & & $\mathrm{M}$ & & A \\
\hline & Postrera & & & & M & & & & $\mathrm{M}$ & $\mathbf{M}$ \\
\hline \multirow[t]{2}{*}{6} & Primera & & & & M & & & & $\mathrm{M}$ & A \\
\hline & Postrera & & & & M & & & & $\mathrm{M}$ & $\mathrm{M}$ \\
\hline
\end{tabular}

producción de leña. La parcela en abonera, en cambio genera un flujo de beneficios constante a lo largo de los seis años. Al finalizar el ciclo el agricultor quema la abonera y el ciclo se repite.

\section{Costos que varían de los sistemas}

Los presupuestos se confeccionan para la tecnología dominante en 1991 de acuerdo a los datos provistos por la encuesta realizada por la SRN y CIMMYT en 1992 (Buckles et al.,1992) Los coeficientes técnicos y los precios de insumos, mano de obra y servicios utilizados fueron también los predominantes en ese año a nivel de agricultor y se obtuvieron mediante una encuesta informal especialmente diseñada y ejecutada para ese propósito por la SRN y el CYMMYT en 1992. En el Cuadro 5 se listan los valores de las necesidades de mano de obra y otros coeficientes técnicos usados en la confección de los presupuestos parciales. 
Cuadro 5. Coeficientes técnicos usados en la construcción de los costos de producción de maíz en el Litoral Atlántico de Honduras.

\begin{tabular}{lr}
\hline Actividades & Unidades \\
\hline Chapia/quema Guamil & $20,0 \mathrm{jor} / \mathrm{ha}$ \\
Chapia/quema Guatal & $15,0 \mathrm{jor} / \mathrm{ha}$ \\
Chapia residuo primera & $12,0 \mathrm{jor} / \mathrm{ha}$ \\
Chapia de abonera & $10,0 \mathrm{jor} / \mathrm{ha}$ \\
Semilla maíz & $14,5 \mathrm{~kg} / \mathrm{ha}$ \\
Mano de obra siembra maíz & $5,0 \mathrm{jor} / \mathrm{ha}$ \\
Semilla frijol de abono & $14,0 \mathrm{~kg} / \mathrm{ha}$ \\
Mano de obra siembra frijol de abono & $5,0 \mathrm{jor} / \mathrm{ha}$ \\
Primera limpia manual primavera & $12,5 \mathrm{jor} / \mathrm{ha}$ \\
Primera limpia manual postrera & $11,0 \mathrm{jor} / \mathrm{ha}$ \\
Primera limpia manual post. an abonera & $9,0 \mathrm{jor} / \mathrm{ha}$ \\
Primera limpia manual con abonera & $9,0 \mathrm{jor} / \mathrm{ha}$ \\
Gramoxone, segundo control & $1,25 \mathrm{l} / \mathrm{ha}$ \\
Aplicación de gramoxone & $2,5 \mathrm{jor} / \mathrm{ha}$ \\
Dobla del maíz & $5,0 \mathrm{jor} / \mathrm{ha}$ \\
Cosecha & $10,0 \mathrm{jor} / \mathrm{ha}$ \\
Acarreo & $16,0 \mathrm{jor} / \mathrm{ha}$ \\
\hline
\end{tabular}

Para el cálculo de los costos que varían entre ambos sistemas se reconocen dos períodos: Un primer año de implantación y cinco años subsecuentes de producción para completar los seis años del ciclo medio de la abonera. El Cuadro 6 muestra los costos que varían el año de implantación de la abonera mientras que el Cuadro 7 presenta los costos en un año de producción. A partir del segundo año el ciclo de costos del sistema de abonera se repite cada año a lo largo de la vida útil promedio de la abonera.

A continuación se describen los principales rubros que componen el flujo de costos tanto para el año de implantación como para aquellos de producción:

\section{i) Preparación del terreno}

En el año de implantación, la estimación de los costos de preparación del terreno considera que el agricultor chapea un guamil de cinco o más años edad para la siembra de primavera (ciclo tradicional) o en la postrera para la implantación de la abonera. La mayoría de los agricultores $(60 \%)$ comienzan las aboneras en parcelas que han permanecido en descanso por un período de aproximadamente 6 años.

Posteriormente, durante el período de producción, la preparación del terreno se facilita en el sistema de abonera ya que la chapea del frijol de abono requiere un número significativamente menor de jornales que el sistema tradicional.

\section{ii) Siembra}

En lo que a la siembra de maíz se refiere, las diferencias de costos, entre ambos sistemas, se atribuyen a la diferencia en los costos de oportunidad de la semilla de maíz debido a la estacionalidad de 
Cuadro 6. Costos de producción de maíz en una parcela el año de implantación de la abonera.

Parcela usada para sembrar maíz bajo dos sistemas (L/ha):

En abonera

Tradicional

Maíz de primavera

\section{Preparación terreno}

1. Chapia y quema

Siembra

4. Semilla ${ }^{1}$

5. Mano de obra siembra

Control de malezas

6. Primer control manual

7. Paraquat

19,9

Aplicación paraquat

Subtotal maíz primavera

200,5

\section{Maíz de postrera}

\section{Preparación terreno}

1. Chapia

Siembra de maíz

4. Semilla ${ }^{1}$

5. Mano de obra siembra

Control de malezas

6. Primer control manual

7. Paraquat

19,9

19,9

Aplicación paraquat

Siembra frijol de abono

Semilla $^{1} \quad 9,9$

Mano de obrs siembra 21,9

Subtotal maíz de postrera

$\begin{array}{lll}\text { TOTAL AÑO IMPLANTACION } \quad 222,8 & 356,6\end{array}$

El precio de la semilla de maíz se toma como el costo de oportunidad del precio del maíz en noviembre-diciembre para postrera y junio-julio para primavera resultando en 0.32 y $0.52 \mathrm{~L} / \mathrm{kg}$ respectivamente. El preciode la semilla de frijol de abono en el área es de $0.71 \mathrm{~L} / \mathrm{kg}$. 
Cuadro 7. Costos que varían en un año de producción de maíz bajo el sistema tradicional y de abonera en el Litoral Atlántico.

Parcela usada para sembrar maíz bajo dos sistemas:

En abonera Tradicional

\section{Maíz de primavera}

\section{Preparación terreno}

1. Chapia y quema

\section{Siembra}

4. Semilla

5. Mano de obra siembra

Control de malezas

6. Primer control manual

7. Paraquat

Aplicación paraquat

\section{Maíz de postrera}

\section{Preparación terreno}

1. Chapia 43,8

Siembra de maíz

4. Semilla $\quad 4,6$

5. Mano de obra siembra 21,9

Control de malezas

6. Primer control manual 48,2

7. Paraquat $\quad 19,9$

Aplicación paraquat $\quad 8,8$

Subtotal maíz de postrera $\quad 147,2$

$\begin{array}{lll}\text { TOTAL AÑO DE PRODUCCION } \quad 147,2 & 165,5\end{array}$

los precios de este producto. En el caso del frijol de abono, solo se considera el costo de semilla y siembra del frijol en el año de implantación, no se consideran costos de siembra en los años de producción ya que una vez establecida la abonera la mayor parte de los agricultores dejan que el frijol de abono se resiembre.

\section{iii) Control de malezas}

Esta es otra operación donde existen diferencias entre sistemas debido en primer lugar a que las malezas son más agresivas en primavera que en postrera, y en segundo porque la abonera ejerce un control sobre el desarrollo de las malezas.

Se considera que en el año de implantación, no 
existen diferencias entre el maíz de postrera con y sin abonera, dado que el frijol de abono se siembra cuando ya han ocurrido los dos controles que normalmente se realizan en el cultivo. Sin embargo, a partir del segundo año el control de malezas en el maíz en abonera requiere menos mano de obra que el maíz sin abonera debido al control natural del frijol de abono. La abonera implica un ahorro en los costos para el control de malezas de aproximadamente un $10 \%$ respecto al control requerido por el maíz sin abonera (Cuadro 6).

\section{Beneficios brutos de los sistemas}

El Cuadro 8 presenta los promedios de los rendimientos obtenidos por los agricultores en sus parcelas de maíz durante la postrera y primavera de acuerdo a tres fuentes de información. Aún cuando se observa una considerable variación entre ellas, es posible observar que existe cierta consistencia en las diferencias entre sistemas (Cuadro 9). Los rendimientos de el maíz en abonera superan en todos los casos a los rendimientos medios del maíz bajo los demás sistemas. Los rendimientos del maíz de primavera supera a aquellos obtenidos por el maíz de postrera cultivado sin abonera.
Para calcular el flujo de rendimientos se debe considerar que el rendimiento del maíz en abonera es dependiente de la edad que tenga este sistema. Se espera que en el primer año o sea el año de implantación del frijol de abono, los rendimientos de maíz no se vean afectados significativamente ya que la mayoría de los agricultores siembra el frijol de abono a la floración del maíz. Aunque es probable que los rendimientos aumenten en alguna medida el segundo año de sembrado el frijol de abono, no es hasta el tercer año en que los agricultores afirman que las aboneras comienzan a producir más. Tomando los promedios ponderados del Cuadro 8 como base, y asumiendo que los rendimientos de maíz no se ven afectados en los dos primeros años de implantada la abonera se obtiene el flujo anual de rendimientos bajo los sistemas alternativos tal como se muestra en el Cuadro 10.

En el caso del sistema tradicional, se estimó que después de tres años y medio de descanso el guamil produce un promedio de 188 cargas de leña por hectárea (una carga tiene 100 leños).

Para obtener el flujo de beneficios brutos se ponderan los rendimientos por el precio de campo de los productos que en este caso consisten de: maíz de primavera, maíz de postrera, y leña en el caso del sistema tradicional.

Cuadro 8. Rendimientos promedios del maíz cultivados bajo diferentes sistemas de acuerdo a tres sondeos en el área del Litoral Atlántico de Honduras ${ }^{1}$

\begin{tabular}{|c|c|c|c|}
\hline \multirow[b]{2}{*}{ Fuente } & \multicolumn{2}{|c|}{$\begin{array}{c}\text { Postrera } \\
\text { kg/ha. }\end{array}$} & \multirow[t]{2}{*}{$\begin{array}{c}\text { Primavera } \\
\text { kg/ha. }\end{array}$} \\
\hline & C/abonera & S/abonera & \\
\hline Sondeo 1989 & $2622(23)$ & $1472(11)$ & \\
\hline Sondeo 1991 & $2638(9)$ & $835(7)$ & $1668(2)$ \\
\hline Sondeo 1992 & $2340(8)$ & $1413(7)$ & \\
\hline Encuesta 1992 & $1373(66)$ & $929(49)$ & $1200(49)$ \\
\hline Promedio ponderado $^{2}$ & 1824 & 1047 & 1218 \\
\hline
\end{tabular}


Cuadro 9. Cambios absolutos y relativos del rendimiento de maíz bajo diferentes sistemas de cultivo.

\begin{tabular}{|c|c|c|c|c|c|c|}
\hline \multirow[t]{4}{*}{ Fuentes de información } & \multicolumn{6}{|c|}{$\begin{array}{l}\text { Diferencias absolutas y relativas del rendimiento de maíz } \\
\text { obtenido bajo diferentes sistemas de cultivo }\end{array}$} \\
\hline & \multicolumn{4}{|c|}{ Maíz en abonera respecto a: } & \multicolumn{2}{|c|}{ Maíz sin abonera respecto a } \\
\hline & \multicolumn{2}{|c|}{ Sin abonera } & \multicolumn{2}{|c|}{ Primavera } & \multicolumn{2}{|c|}{ Primavera } \\
\hline & $\mathrm{kg} / \mathrm{ha}$ & $\%$ & $\mathrm{~kg} / \mathrm{ha}$ & $\%$ & $\mathrm{~kg} / \mathrm{ha}$ & $\%$ \\
\hline Sondeos & 1293 & 51 & 865 & 34 & -428 & -34 \\
\hline Encuesta 92 & 396 & 34 & 121 & 10 & -275 & -35 \\
\hline
\end{tabular}

Cuadro 10. Flujo estimado de rendimiento de maíz cultivado bajo tres sistemas en el Litoral Atlántico de Honduras.

\begin{tabular}{|c|c|c|c|}
\hline \multirow[t]{3}{*}{ Años } & \multicolumn{3}{|c|}{ Rendimiento de maíz en $\mathrm{Kg} / \mathrm{Mz}$. } \\
\hline & \multicolumn{2}{|c|}{ Postrera } & \multirow[t]{2}{*}{ Primavera $^{1}$} \\
\hline & En abonera & Sin abonera & \\
\hline 1 (implantación) & 1047 & 1047 & 1218 \\
\hline 2 & 1047 & & 1218 \\
\hline 3 al 6 & 1824 & & \\
\hline
\end{tabular}

1. Se debe considerar que para sostener el rendimiento de maíz por un periodo de 6 años sin abonera se debe cultivar una nueva parcela cada 1.5 años. Para más detalles vease el texto.

Dada la naturaleza de, los sistemas que se evaluan es necesario obtener precios de tendencia o de largo plazo de los productos. En los Cuadro 11 y 12 se muestra la evolución de los precios reales a nivel de agricultor del maíz, arroz y frijol común durante el período 1980-1991. Dado que a partir de 1988 el Gobierno de Honduras, cambia la política económica dentro de un programa de ajuste estructural y comienza un ajuste de precios relativos, se toma como precio de largo plazo del maíz el promedio de los precios durante el período 1987-1991.

Este precio refleja sin embargo un promedio anual y debe ser ajustado para tener en cuenta la estacionalidad predominante en los precios del maíz. El exceso estacional de oferta hace caer los precios al por mayor durante los meses de octubre, noviembre y diciembre, época de cosecha de la primavera. A partir de enero los precios comienzan a subir hasta alcanzar un pequeño pico en abril, a partir de ese momento descienden a medida que ocurre la salida de la cosecha de postrera en mayojunio pero de todas maneras se mantienen por arriba del promedio anual (Figura 5). Este ciclo estacional a nivel de precios al mayorista se repite a nivel de agricultor. Aunque no se dispone de una serie de precios mensuales tan completa como aquella de los precios a nivel de mayorista, la estacionalidad de los precios a nivel de finca para el período 1990-1991 presenta el mismo patrón estacional que a nivel mayorista con los precios de postrera superando al promedio en $23 \%$ mientras que para la siembra de primavera se encuentran un $25 \%$ por debajo.

Una vez estimados los precios de tendencia ajustados por la estacionalidad se estima el precio de campo del maíz el cual se define como el precio de venta, menos el total de costos proporcionales al rendimiento (CIMMYT). 
Cuadro 11. Evolución de los precios reales del maíz, arroz y frijol a nivel de agricultor durante el período 19801991.

\begin{tabular}{lcccccc}
\hline \multirow{2}{*}{ Años } & \multicolumn{3}{c}{ Precios reales (L/kg) } & & \multicolumn{2}{c}{ Precios relativos } \\
\cline { 2 - 3 } & Maíz & Arroz & Frijol & & Pa/Pm & Pf/Pm \\
\hline 1980 & 0,35 & 1,16 & 0,93 & & 3,31 & 2,65 \\
1981 & 0,31 & 1,14 & 0,77 & & 3,70 & 2,48 \\
1982 & 0,33 & 1,11 & 0,78 & & 3,34 & 2,36 \\
1983 & 0,33 & 1,07 & 0,76 & & 3,26 & 2,32 \\
1984 & 0,28 & 0,94 & 0,74 & & 3,34 & 2,64 \\
1985 & 0,32 & 0,93 & 0,77 & & 2,95 & 2,46 \\
1986 & 0,33 & 0,89 & 0,67 & & 2,72 & 1,98 \\
1987 & 0,33 & 0,48 & 0,66 & & 1,44 & 3,42 \\
1988 & 0,32 & 0,46 & 1,08 & & 1,47 & 2,27 \\
1989 & 0,32 & 0,51 & 0,72 & & 1,61 & 3,24 \\
1990 & 0,36 & 0,62 & 1,16 & & 1,72 & 2,44 \\
1991 & 0,76 & 0,90 & 1,85 & & 1,18 & 2,67 \\
Tendencia & 0,42 & 0,59 & 1,09 & 1,48 & \\
\hline
\end{tabular}

Fuente: Secretaría de Recursos Naturales, Unidad de Planificación Sectorial Agrícola, Departamento de Información Agrícola. Compendio Estadístico Agropecuario 1991. Tegucigalpa, Honduras 1991.

Precios deflacionados por el Indice General de Precios al Consumidor. Base: 1985. Los precios de tendencia se calcularon como el promedio de los precios reales en el período 1987-1991.

Cuadro 12. Evolución y tendencia de los precios reales de los insumos y servicios más importantes empleados en la producción de maíz en el Litoral Atlántico de Honduras 1980-1991.'

\begin{tabular}{|c|c|c|c|c|}
\hline \multirow[t]{2}{*}{ Años } & \multicolumn{4}{|c|}{ Insumos } \\
\hline & $\begin{array}{c}\text { Nitrógeno }^{2 /} \\
\text { L/kg }\end{array}$ & $\begin{array}{c}\text { Semilla }^{3 /} \\
\text { mejorada L/kg }\end{array}$ & $\begin{array}{c}\text { Paraquat } \\
\text { L/lt }\end{array}$ & $\begin{array}{c}\text { Mano de obra } \\
\text { L/jor. }\end{array}$ \\
\hline 1980 & 1,90 & 1,38 & 15,11 & 7,80 \\
\hline 1981 & $\cdot 1,99$ & 1,26 & 20,53 & 7,34 \\
\hline 1982 & 1,70 & 1,23 & 19,60 & 6,18 \\
\hline 1983 & 1,60 & 1,19 & 17,22 & 5,85 \\
\hline 1984 & 1,46 & 1,14 & 15,57 & 5,34 \\
\hline 1985 & 1,30 & 1,10 & 14,26 & 5,00 \\
\hline 1986 & 1,24 & 1,05 & 13,70 & 5,51 \\
\hline 1987 & 1,05 & 1,18 & 12,38 & 5,24 \\
\hline 1988 & 1,00 & 1,18 & 11,84 & 6,40 \\
\hline 1989 & 1,14 & 1,08 & 14,66 & 3,98 \\
\hline 1990 & 1,43 & 1,45 & 19,06 & 3,49 \\
\hline 1991 & 1,92 & 2,16 & 21,52 & 2,77 \\
\hline Tendencia & 1,31 & 1,41 & 15,89 & 4,38 \\
\hline
\end{tabular}

1 Precios deflacionados por el Indice General de Precios al Consumidor. Base: 1985. Los precios de tendencia se calcularon como el promedio de los precios reales en el período 1987-1991.

2 Nitrógeno de la urea.

3 Basado en el precio de la variedad mejorada Sintético Tuxpeño.

4 L = Lempiras 


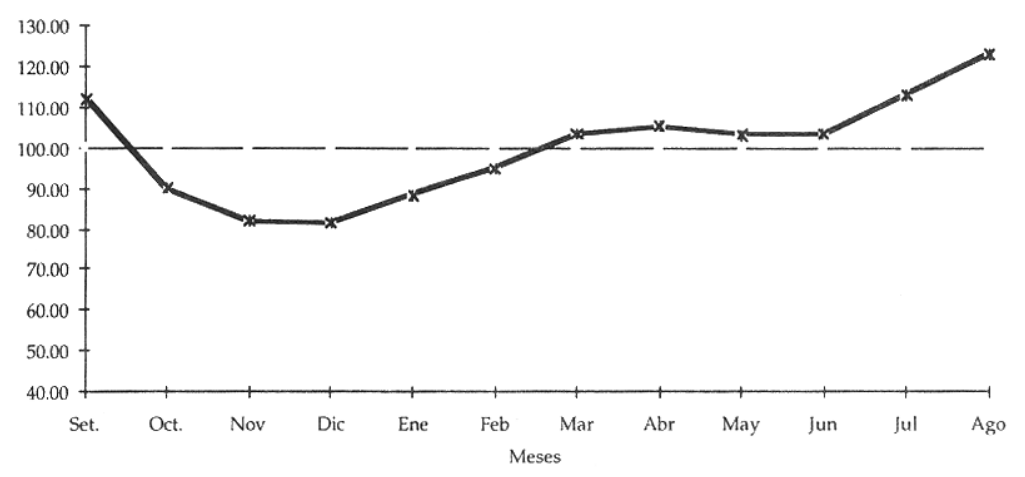

Figura 5. Indice estacional de los precios del maíz a nivel mayorista durante el periodo 1970-1991, Honduras.

El Cuadro 13 muestra, en su primera línea, los precios de venta de tendencia para el maíz de postrera y de primavera. Para obtener el precio de campo se sustraen de estos precios los costos proporcionales al rendimiento como son aquellos de dobla, cosecha, desgrane y acarreo. Algunos de estos costos difieren entre sí de acuerdo al sistema que se trate, por ejemplo en primera el maíz se dobla mientras que en postrera no. También, la cosecha requiere más mano de obra si se trata de una abonera. La última fila del Cuadro 14 muestra los precios de campo de largo plazo encontrados. Para fines del cálculo del flujo de beneficios en la próxima sección se toma $0.44 \mathrm{~L} / \mathrm{kg}$ como precio de largo plazo para ambos sistemas en postrera.

En el caso de la leña, no fué posible obtener una serie temporal de precios de la carga de leña para así obtener un precio de tendencia, por lo que se toma el promedio de los precios reales de los años 1990 y 1991 que resulta en $\mathbf{3 . 6 5}$ L/carga, para calcular los beneficios brutos de este subproducto del sistema tradicional.

\section{Rentabilidad de la abonera: retornos al capital}

Varios indicadores pueden ser utilizados para evaluar la rentabilidad de largo plazo de los sistemas en términos de los retornos al capital (Harrington). En este trabajo se emplea el valor presente neto (VPN) del flujo incremental de beneficios netos de las alternativas (Steiner, 1980). El VPN no es más que la diferencia entre el valor descontado de beneficios menos el valor descontado de los costos. Si el valor calculado del VPN del flujo incremental es mayor que cero, significa que los beneficios descontados son mayores que los costos por lo que la alternativa es economicamente viable.

El Cuadro 14 muestra la evolución, para ambos sistemas, de los flujos anualesde costos que varían, donde se ha incluido el costo de cosecha, confección y acarreo de las cargas de leña en el sexto año del sistema tradicional, como así también el flujo de los beneficios brutos anuales el cual sigue el patrón de los rendimientos de maíz a excepción del último año en donde se considera el valor de la producción de leña como un subproducto del sistema tradicional.

Sustrayendo el patrón de costos anuales de los beneficios brutos, se obtiene el flujo anual de beneficios netos para ambas alternativas (Cuadro 14). Se observa que con excepción del primer año (implantación), los beneficios netos del sistema de abonera superan a los obtenidos por el sistema tradional.

El Cuadro 14 presenta también en su última columna, el flujo de beneficios netos incrementales. Como el uso de la tierra en abonera ahorra costos al comienzo del ciclo, el incremento se estima sustrayendo los beneficios netos del 
Cuadro 13. Precio de venta, costos proporcionales al rendimiento, y precio de venta para el maíz bajo tres formas de siembra en el Litoral Atlántico de Honduras.

\begin{tabular}{lcccc}
\hline & \multicolumn{2}{c}{ Precio s y costos proporcionales al rendimiento (L/kg) } \\
\cline { 2 - 3 } & \multicolumn{2}{c}{ Postrera } & & Primavera \\
\cline { 2 - 3 } & En abonera & Sin abonera & & Primavera \\
\hline 1- Precio de venta & 0,52 & 0,52 & 0,32 \\
2- Costos proporcionales & 0,093 & 0,079 & 0,094 \\
Dobla & 0,034 & & 0,015 \\
Cosecha & 0,039 & 0,02 & 0,02 \\
Acarreo & 0,02 & 0,039 & 0,039 \\
Desgrane & 0,43 & 0,02 & 0,02 \\
3- Precio de campo & & 0,44 & 0,23 \\
\hline
\end{tabular}

Cuadro 14. Flujo de costos, beneficios brutos, beneficios netos por sistema e incrementales durante los seis años del ciclo de abonera.

\begin{tabular}{|c|c|c|c|c|c|c|c|c|}
\hline \multirow[t]{2}{*}{ Años } & \multirow[t]{2}{*}{ Ciclos } & \multicolumn{3}{|c|}{ Abonera } & \multicolumn{3}{|c|}{ Tradicional } & \multirow{2}{*}{$\frac{\text { BNt-a }}{\text { Incremental }}$} \\
\hline & & $\begin{array}{l}\text { Costos } \\
\text { (L/ha) }\end{array}$ & $\begin{array}{c}\text { BB } \\
\text { (L/ha) }\end{array}$ & $\begin{array}{c}\text { BN } \\
(\mathbf{L} / \mathbf{h a})\end{array}$ & $\begin{array}{l}\text { Costos } \\
\text { (L/ha) }\end{array}$ & $\begin{array}{c}\text { BB } \\
(\mathrm{L} / \mathrm{ha})\end{array}$ & $\begin{array}{c}\text { BN } \\
(\mathrm{L} / \mathrm{ha})\end{array}$ & \\
\hline & Inicial & 0 & 0 & 0 & 200,5 & 0 & $-200,5$ & $-200,5$ \\
\hline \multirow[t]{2}{*}{1} & 1 & 222,8 & 0 & $-222,8$ & 156,1 & 280,1 & 124 & 346,8 \\
\hline & 2 & 0 & 460,7 & 237,9 & 165,5 & 460,7 & 295,2 & 57,3 \\
\hline \multirow[t]{2}{*}{2} & 3 & 147,7 & 0 & $-147,7$ & 0 & 280,1 & 280,1 & 427,8 \\
\hline & 4 & 0 & 460,7 & 460,7 & 0 & 0 & 0 & $-460,7$ \\
\hline \multirow[t]{2}{*}{3} & 5 & 147,7 & 0 & $-147,7$ & 0 & 0 & 0 & 147,7 \\
\hline & 6 & 0 & 802,6 & 802,6 & 0 & 0 & 0 & $-802,6$ \\
\hline \multirow[t]{2}{*}{4} & 7 & 147,7 & 0 & $-147,7$ & 0 & 0 & 0 & 147,7 \\
\hline & 8 & 0 & 802,6 & 802,6 & 0 & 0 & 0 & $-802,6$ \\
\hline \multirow[t]{2}{*}{5} & 9 & 147,7 & 0 & $-147,7$ & 0 & 0 & 0 & 147,7 \\
\hline & 10 & 0 & 802,6 & 802,6 & 0 & 0 & 0 & $-802,6$ \\
\hline \multirow[t]{2}{*}{6} & 11 & 147,7 & 0 & $-147,7$ & 0 & 0 & 0 & 147,7 \\
\hline & 12 & 0 & 802,6 & 802,6 & 234,6 & 684,4 & 449,8 & $-352,8$ \\
\hline
\end{tabular}


sistema tradicional de aquellos que se obtendrían usando la tierra en abonera. De esa manera la última columna del Cuadro 14 representa el flujo de beneficios netos adicionales que resultarían de usar la tierra bajo el sistema tradicional en vez de usarla bajo el sistema de abonera.

El Cuadro 15 presenta los valores encontrados del VPN para diferentes años de vida de la abonera. Para el cálculo del VPN se toman como base dos tasas de descuento extremas: una tasa de descuento del $40 \%$ que representa aproximadamente el costo real del dinero para los agricultores del área 2 y una tasa del $10 \%$ que refleja el interés real de los préstamos al sector agropecuario durante el período 1984-19883. Se realiza además un análisis a una tasa intermedia del $20 \%$.

Los resultados muestran que, independientemente de la tasa de descuento que se use, a partir del segundo año el uso de la tierra para cultivar maíz en postrera bajo el sistema tradicional no resulta rentable. Esta alta rentabilidad del sistema de abonera estaría explicando parcialmente la rápida difusión del sistema en la región. Sin embargo, el sistema de abonera requiere de un período de inversión inicial de un año durante el cual el sistema tradicional de uso de la tierra sí resulta más atractivo. Es decir que para aquellos de agricultores que solo consideren usar la tierra un solo año (dos ciclos) como podrían ser arrendatarios o otras formas precarias de cultivar la tierra, no les resulta rentable instalar la abonera.

El análisis de los retornos al capital es un indicador parcial de la rentabilidad de una tecnología, existen otras medidas de la rentabilidad que pueden ser más relevantes para pequeñas fincas, como es el cálculo del retorno a la mano de obra familiar (Low, Barlett). Dvorak (1993) muestra la importancia de la mano de obra en la economía de la agricultura migratoria entre agricultotores pequeños. Por este motivo y dada la percepción de los agricultores sobre la importancia del ahorro de mano de obra del sistema de abonera sobre el tradicional en la próxima sección se examina más en detalle el impacto del sistema

Cuadro 15. Indicadores de la rentabilidad relativa del sistema de siembra de maíz en abonera. Litoral Atlántico, Honduras 1991.

\begin{tabular}{lccc} 
Años de Abonera & \multicolumn{3}{c}{$\begin{array}{c}\text { Valor Presente Neto (lempiras) } \\
\text { (Estimado a tres tasas de descuento anuales) }\end{array}$} \\
\cline { 2 - 4 } & $\mathbf{1 0 \%}$ & $\mathbf{2 0 \%}$ & $\mathbf{4 0 \%}$ \\
\hline 1 & 182 & 162 & 128 \\
2 & -36 & -47 & -72 \\
3 & -311 & -192 & -56 \\
4 & -749 & -491 & -201 \\
5 & -1147 & -738 & -302 \\
6 & -1257 & -799 & -322 \\
\hline
\end{tabular}

2 Se estima una tasa de interés anual promedio de $75 \%$ menos un $35 \%$ de inflación.

3 Hasta 1987, la tasa real anual de interés se mantuvo estable alrededor de 6 a $8 \%$ anual, pero a partir de 1988 la tasa anual de crecimiento de la inflación supera a una estancada tasa de interés nominal con lo cual las tasas reales declinan hasta hacerse negativas en los dos últimos años del período. Dado que estas últimas tasas no son sostenibles y resultan de un descontrol coyuntural de las tasas de inflación, no se consideran en el cálculo de la tasa real promedio. 
de abonera sobre el patrón de uso de la mano de obra en la finca y se estiman los retornos sobre la mano de obra familiar bajo los dos sistemas.

\section{Rentabilidad de la abonera: retornos a la mano de obra.}

La introducción de la abonera en el. sistema de finca modifica no solamente la forma de producción de maíz de postrera sino que implica una reacomodación del uso de tierra y de la mano de obra en la finca. El aumento en la superficie cultivada con maíz en postrera es posible porque la abonera una vez en producción requiere poco uso de mano de obra en la preparación del terreno.

Si se consideran las necesidades de mano de obra para cultivar maíz con abonera con aquellas requeridas para cultivar sin abonera o para el maíz en primavera en un año determinado (producción) las diferencias muestran que el sistema de abonera representa un ahorro del $8 \%$ respecto al maíz en postrera y de $40 \%$ respecto al maíz de primavera cuando se necesite abrir un guamil para sembrarlo. La eficiencia de uso de tierra y mano de obra del sistema de abonera se pone en evidencia si se considera no un año aislado pero el ciclo completo de vida de la abonera de 6 años. En este ciclo la abonera no solo permite un aumento en el área cosechada de 50\% con un aumento de solo $25 \%$ en el número de parcelas usadas sino que requiere un aumento de 183 jornales que representa un aumento del $45 \%$ sobre el total de mano de obra requerida por el sistema tradicional.

La Figura 6 muestra los requerimientos mensuales de mano de obra total y por hectárea cultivada en una finca que cultiva maíz sin abonera comparada con una finca con abonera. A pesar que esta última tiene una superficie cultivada mayor en $28 \%$ no se registran diferencias significativas en términos de los requerimientos totales a excepción del més de julio cuando se superponen las limpias del maíz de primavera, del arroz y la cosecha del frijol de mayo. Noviembre es otro més que representa altos requerimientos de mano de obra debido principamente a las cosechas y acarreo del maíz de primavera, del arroz, del control de malezas del frijol de octubre y de la chapia para la siembra de frijol de febrero. En términos de los requerimientos por unidad de tierra, la finca con abonera representa un importante ahorro de mano de obra en el crítico mes de noviembre y en diciembre.

La Figura 7 muestra la distribución de frecuencia de la disponibilidad de mano de obra en las fincas del Litoral Atlántico en términos de unida des de trabajo por més. Para el cálculo se usó la convención de la FAO donde se adjudica 1 unidad a un adulto varón, 0,7 de unidad a una mujer adulta, 0,50 a un adolescente o una persona mayor independiente de su género y 0,25 de unidad a un niño entre 11 y 15 años independiente del género (Murphy and Sprey). La distribución se centra en un valor de 69 UT/mes y se toma ese valor para determinar a julio y noviembre como los meses críticos respecto a mano de obra en ambos sistemas.

Los retornos a la mano de obra familiar, se estiman como la relación entre el valor presente de los beneficios netos por unidad de mano de obra familiar utilizada en la producción de maíz bajo los dos sistemas alternativos. En este caso los costos que varían no contabilizan aquellos generados por el uso de la mano de obra familiar pero si incorporan el costo del capital en efectivo. Dado que las fincas del área usan una combinación de mano de obra familiar y contratada para las labores de preparación del terreno y siembra se asume, para fines del cálculo, que esas labores se realizan con una proporción de 50\% de mano de obra familiar y $50 \%$ con mano de obra contratada. Las restantes actividades se realizan con mano de obra familiar. El costo del capital se toma como $40 \%$ anual.

El Cuadro 16 presenta la tabla de retornos netos sobre la mano de obra familiar bajo ambos sistemas. Los resultados bajo este criterio confirman aquellos obtenidos mediante el cálculo de los retornos al capital: después de un año inicial de inversión la abonera se vuelve más rentable que el sistema tradicional al segundo año y así permanece bajo todo el período. Más aún, bajo este criterio la abonera y el sistema tradicional rinden igual aún en el año de implantación. 
(a) Totales

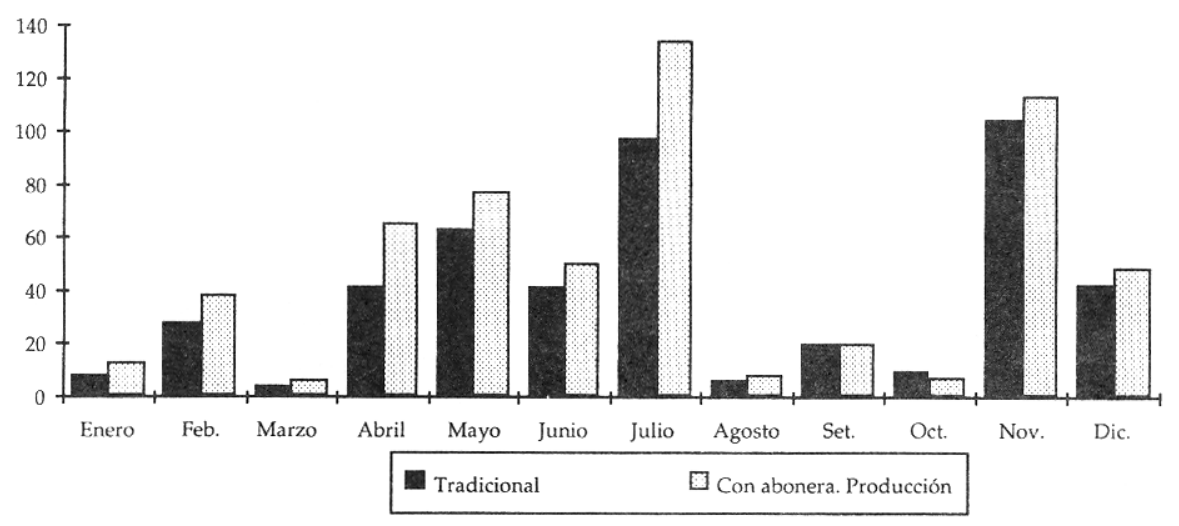

(b) Por hectárea

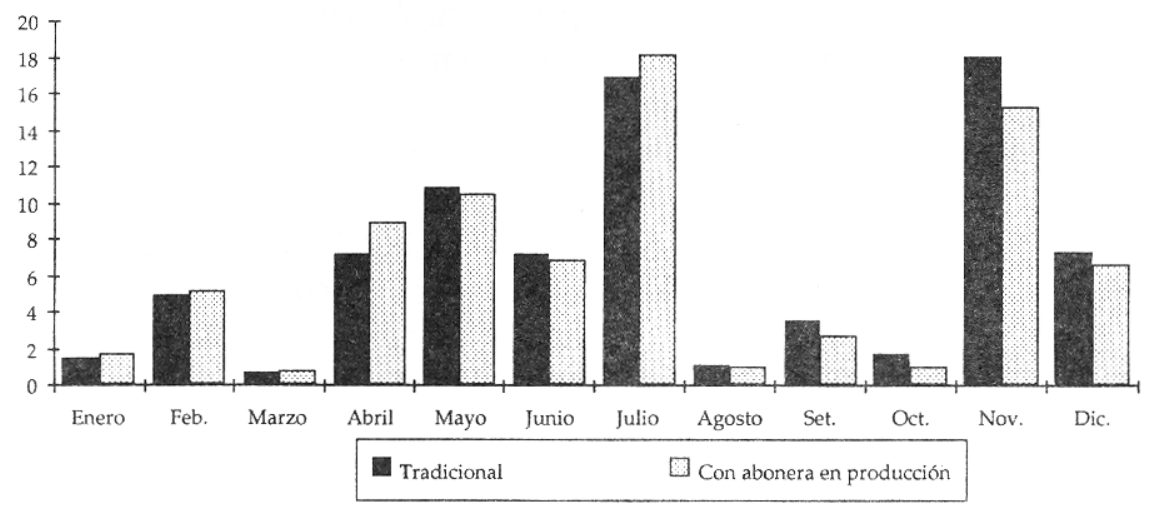

Figura 6. Requerimientos mensuales de mano de obra total por hectárea a nivel de finca.

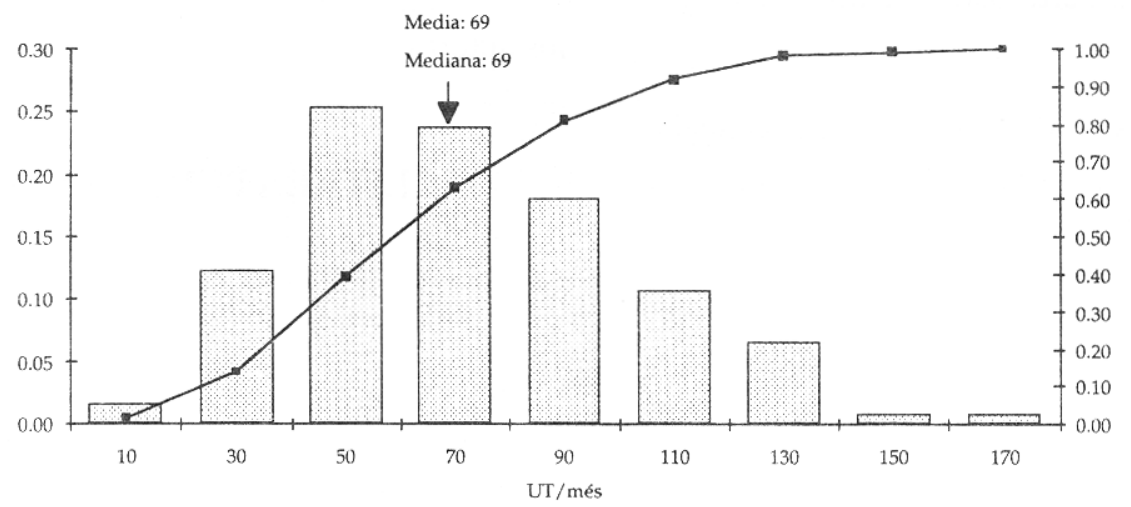

Figura 7. Distribución de la disponibilidad mensual de mano de obra en las fincas del Litoral Atlántico 
Cuadro 16. Tabla de retornos netos a la mano de obra familiar en lempiras por jornal.

\begin{tabular}{|c|c|c|c|c|c|c|}
\hline \multirow[t]{2}{*}{ Años de abonera } & \multicolumn{3}{|c|}{ Sistema con abonera } & \multicolumn{3}{|c|}{ Sistema tradicional } \\
\hline & $10 \%$ & $20 \%$ & $40 \%$ & $10 \%$ & $20 \%$ & $40 \%$ \\
\hline 1 & 12,2 & 12,2 & 12,2 & 11,7 & 11,7 & 11,7 \\
\hline 2 & 14,4 & 13,4 & 12,8 & 10,6 & 10,4 & 10,0 \\
\hline 3 & 18,9 & 17,1 & 14,4 & 10,6 & 10,4 & 10,0 \\
\hline 4 & 20,7 & 17,9 & 14,1 & 10,6 & 10,4 & 10,0 \\
\hline 5 & 21,4 & 17,8 & 13,2 & 10,6 & 10,4 & 10,0 \\
\hline 6 & 21,5 & 17,2 & 12,2 & 10,5 & 9,4 & 7,8 \\
\hline
\end{tabular}

Nótese también que para las tres tasas de descuento los retornos a la mano de obra familiar bajo ambos sistemas superan holgadamente al salario real de largo plazo de 4,38 L/jornal.

\section{El impacto del sistema de abonera sobre el valor de la tierra}

La introducción del sistema de abonera en el sistema de finca modifica significativamente el valor de la tierra una vez que se encuentra establecido en un área, y que los agricultores reconocen las bondades del sistema en la generación de una mayor rentabilidad del cultivo. Este reconocimiento se expresa en el área del Litoral Atlántico a través de la disposición a pagar mejores precios por el alquiler de la tierra para el cultivo del maíz de postrera.

La información suministrada por los sondeos realizados en el área permitieron identificar una diferencia significativa entre el precio del alquiler de la tierra para la siembra de maíz en postrera si es que la parcela estaba o no en abonera (Cuadro 17). de acuerdo a los valores más frecuentes (moda) encontrados los agrciultores estarían dispuestos a pagar un sobreprecio de 50 lempiras/ha por el derecho de cultivar maíz en una parcela en abonera en vez de hacerlo en una que ha estado en descanso. Este sobreprecio refleja la percepción de los agricultores de la mejor capacidad productiva de la tierra que se encuentra bajo el sistema de abonera. Este valor es bastante inferior a las diferencias entre beneficios netos que se obtienen con ambos sistemas que equivale aproximadamente a 350 lempiras por hectárea cuando la abonera tiene más de tres años.

\section{AGRADECIMIENTO}

Los autores desean agradcer a D. Buckles, D. Byerlee, L. Harrington, M. Lopez, M. Morris y R. Tripp por sus comentarios y sugerenecias hechas a una versión previa del presente trabajo. Cualquier error remanente es de la responsabilidad de los autores.

\section{LITERATURA CITADA}

AVILA, R.; J. L. LOPEZ. 1990. Sondeo preliminar en la asociación maíz frijol de abono (Mucuna sp) en el Litoral Atlántico de Honduras. In: Memoria XXXVI Reunión Anual del Programa Cooperativo Centroamericano para el Mejoramiento de Cultivos y Animales (PCCMCA), San Salvador, El Salvador, 26-30 de Marzo de 1990. 
Cuadro 17. Distribución del valor de alquiler de la tierra de acuerdo a a su uso. Litoral Atlántico, Honduras,1991.

\begin{tabular}{|c|c|c|c|c|c|}
\hline & \multicolumn{4}{|c|}{ Alquiler por sistema (L/Ha.) } & \multirow{3}{*}{ Potrero } \\
\hline & \multicolumn{2}{|c|}{ Abonera } & \multirow{2}{*}{$\frac{\text { Guatal }}{\text { Menos } 3 \text { años }}$} & \multirow{2}{*}{$\frac{\text { Guamil }}{\text { Más } 3 \text { años }}$} & \\
\hline & Menos 3 años & Más 3 años & & & \\
\hline Media & 135 & 140 & 83 & 90 & 78 \\
\hline Mediana & 125 & 125 & 75 & 75 & 69 \\
\hline Moda & 125 & 125 & 63 & 63 & 125 \\
\hline Desv. Est. & 33,1 & 32,6 & 43,1 & 41,6 & 39,1 \\
\hline Mínimo & $40 /, 8$ & $60 /, 8$ & $20 /, 8$ & 20 & 20 \\
\hline Máximo & 200 & 200 & 200 & 200 & 125 \\
\hline Observ. & 23 & 23 & 23 & 22 & 10 \\
\hline
\end{tabular}

BARLETT, P. F. 1980. Cost-BenefitAnalysis: A Test of Alternative Methodologies. In: Barlett, P. F. (ed): Agricultural Decision Making: Anthropological Contributions to Rural Development. New York, Academic Press. p.137-160.

BUCKLES, D.; J. SALGADO; H. BOJORQUE; H. ANTUNEZ; L. MEJÍA; H. NOLAZCO; L. DE RAMOS; G. MEDINA; R. MATUTE. 1991. Resultados de la encuesta exploratoria sobre el uso de frijol de abono (Stizolobium deeringianum) en las laderas del Litoral Atlántico de Honduras. In: Programa Regional de Maíz para Centro América y el Caribe. Análisis de los Ensayos Regionales de Agronomía, 1991. Guatemala, Junio, 1991.

BUCKLES, D.; I. PONCE; G. SAIN; G. MEDINA. 1993. Uso y difusión del frijol de abono (Mucuna deeringianum) en las la deras del Litoral Atlántico de Honduras. Agron. Mesoam. 4:15-29.

DVORAK KAREN ANN.1992. ResourceManagement by West African Farmers and the Economic of Shifting Cultivation. Amer. J. Agr. Econ. Aug. 1992,p. 809-815.

FLORES, G.; H. LICONA. 1985. Investigación agrícola en campos de agricultores. El caso de Jutiapa, Honduras. En:
Memoria XXXI Reunión Anual del Programa Cooperativo Centroamericano para el Mejoramiento de Cultivos y Animales (PCCMCA), San Pedro Sula, Honduras, Marzo de 1985.

HARRINGTON, L. W. 1991. Measuring Sustainability: Issues and Alternatives. Paper presented at the 11th Annual AFSRE Symposium, Michigan State University, East Lansing, Michigan, 5-10 October, 1991.

HONDURAS. SECRETARIA DE RECURSOS NATURALES. 1991. Compendio Estadístico Agropecuario. 1990-1991. Unidad de Planificación Sectorial Agrícola, Departamento de Información Agrícola. Tegucigalpa, M. D. C. Honduras.

LOW, A. 1986. Agricultural Development in Southern Africa: Fann Household Theory \& the Food Crisis. London: James Currey.

MURPHY, J.; L. H. SPREY. 1992. Monitoring and Evaluation of Agricultural Change. ILRI, Wageningen, 1982.

STEINER HENRY, M. 1980. Public and Private Investments. Socioeconomic Analysis. John Wiley \& Sons, Inc. New York. 